\title{
ON GENERALIZED STATISTICAL CONVERGENCE OF SEQUENCES OF SETS OF ORDER $\alpha$
}

\author{
B. HAZARIKA AND A. ESI
}

Received 23 April, 2015

\begin{abstract}
In this paper we define generalized statistical convergence for sequences of sets of order $\alpha, 0<\alpha \leq 1$ in sense of Wijsman and study some basic properties of this concept.

2010 Mathematics Subject Classification: 40A35; 40G15

Keywords: statistical convergence, $\lambda$-sequence, Wijsman convergence, Orlicz function
\end{abstract}

\section{INTRODUCTION}

The concept of convergence of sequences of points has been extended by several authors to convergence of sequences of sets. The one of these such extensions considered in this paper is the concept of Wijsman convergence. We shall define Wijsman $\lambda$-statistical convergence for sequences of sets of order $\alpha,(0<\alpha \leq 1)$ and establish some basic results regarding the notions Wijsman $\lambda$-statistical convergence and Wijsman statistical convergence for sequences of sets of $\alpha,(0<\alpha \leq 1)$.

The idea of statistical convergence first appeared, under the name of "almost convergence" in the first edition Zygmund [25] of celebrated monograph of Zygmund [26]. Later, this idea was introduced by Fast [8] and Steinhaus [22] and later was introduced by Schoenberg [21], also independently by Buck [1] and studied various authors (see $[5,9,20])$. Mursaleen [17], introduced the notion $\lambda$-statistical convergence for real sequences. For more details on $\lambda$-statistical convergence we refer to [2] and many others. Over the years and under different names statistical convergence has been discussed in the theory of Fourier analysis, ergodic theory and number theory. In the recent years, generalization of statistical convergence have appeared in the study of strong integral summability and the structure of ideals of bounded continuous functions on Stone-Čech compactification of the natural numbers. 
A real or complex number sequence $x=\left(x_{k}\right)$ is said to be statistically convergent to $L$ if for every $\varepsilon>0$

$$
\lim _{n} \frac{1}{n}\left|\left\{k \leq n:\left|x_{k}-L\right| \geq \varepsilon\right\}\right|=0 .
$$

In this case, we write $S-\lim x=L$ or $x_{k} \rightarrow L(S)$ and $S$ denotes the set of all statistically convergent sequences.

The generalized de la Vallée-Poussin mean is defined by

$$
t_{n}(x)=\frac{1}{\lambda_{n}} \sum_{k \in I_{n}} x_{k}
$$

where $I_{n}=\left[n-\lambda_{n}+1, n\right]$. A sequence $x=\left(x_{k}\right)$ is said to be $(V, \lambda)-$ summable to number $L$ (see [15]) if $t_{n}(x) \rightarrow L$ as $n \rightarrow \infty$. If $\lambda_{n}=n$, then $(V, \lambda)$-summability reduces to $(\mathrm{C}, 1)$-summability.

Mursaleen [17] defined $\lambda$-statistically convergent sequence as follows. A sequence $x=\left(x_{k}\right)$ is said to be $\lambda$ - statistically convergent to the number $\mathrm{L}$ if for every $\varepsilon>0$

$$
\lim _{n \rightarrow \infty} \frac{1}{\lambda_{n}}\left|\left\{k \in I_{n}:\left|x_{k}-L\right| \geq \varepsilon\right\}\right|=0 .
$$

Let $\mathrm{S}_{\lambda}$ denotes the set of all $\lambda$-statistically convergent sequences. If $\lambda_{n}=n$, then $S_{\lambda}$ is the same as $S$.

Let $(X, \rho)$ be a metric space. For any point $x \in X$ and any non-empty subset $A \subset X$, the distance from $\mathrm{x}$ to $\mathrm{A}$ is defined by

$$
d(x, A)=\inf _{y \in A} \rho(x, y) .
$$

Let $(\mathrm{X}, \rho)$ be a metric space. For any non-empty closed subsets $A, A_{k} \subset X(k \in \mathbb{N})$, we say that the sequence $\left(A_{k}\right)$ is Wijsman convergent (see $\left.[23,24]\right)$ to $A$ if $\lim _{k} d\left(x, A_{k}\right)=d(x, A)$ for each $x \in X$. In this case we write $W-\lim A_{k}=A$. The concepts of Wijsman statistical convergence and boundedness for the sequence $\left(A_{k}\right)$ were given by Nuray and Rhoades [18] as follows. Let $(X, \rho)$ be a metric space. For any non-empty closed subsets $A, A_{k} \subset X(k \in \mathbb{N})$, we say that the sequence $\left(A_{k}\right)$ is Wijsman statistical convergent to $A$ if the sequence $\left(d\left(x, A_{k}\right)\right)$ is statistically convergent to $d(x, A)$, i.e., for $\varepsilon>0$ and for each $x \in X$

$$
\lim _{n} \frac{1}{n}\left|\left\{k \leq n:\left|d\left(x, A_{k}\right)-d(x, A)\right| \geq \varepsilon\right\}\right|=0 .
$$

In this case, we write $s t-\lim _{k} A_{k}=A$ or $A_{k} \rightarrow A\left(S^{W}\right)$. The sequence $\left(A_{k}\right)$ is bounded if $\sup _{k} d\left(x, A_{k}\right)<\infty$ for each $x \in X$. The set of all bounded sequences of sets denoted by $L_{\infty}$. For details on Wijsman statistical convergent we refer to [11-14] and many others. 


\section{WIJSMAN $\lambda$-STATISTICAL CONVERGENCE}

In this section, we will define Wijsman $\lambda$-statistical convergence of sequences of sets and will give the relations between Wijsman statistical and Wijsman $\lambda$-statistical convergence of sequences of sets.

Definition 1. Let $(X, \rho)$ be a metric space and $\lambda=\left(\lambda_{n}\right)$ be a non-decreasing sequence of positive numbers such that $\lambda_{n+1} \leq \lambda_{n}+1, \lambda_{1}=1, \lambda_{n} \rightarrow \infty$ as $n \rightarrow \infty$ and $I_{n}=\left[n-\lambda_{n}+1, n\right]$. For any non-empty closed subsets $A, A_{k} \subset X(k \in \mathbb{N})$, we say that the sequence $\left(A_{k}\right)$ is Wijsman $\lambda$-statistical convergent to $A$ if the sequence $\left(d\left(x, A_{k}\right)\right)$ is $\lambda$-statistically convergent to $d(x, A)$, i.e., for $\varepsilon>0$ and for each $x \in X$

$$
\lim _{n} \frac{1}{\lambda_{n}}\left|\left\{k \in I_{n}:\left|d\left(x, A_{k}\right)-d(x, A)\right| \geq \varepsilon\right\}\right|=0 .
$$

In this case, we write $S_{\lambda}^{W}-\lim _{k} A_{k}=A$ or $A_{k} \rightarrow A\left(B_{\lambda}^{W}\right)$.

Example 1. Let $X=\mathbb{R}^{2}$ and the sequence $\left(A_{k}\right)$ is defined as follows:

$$
A_{k}=\left\{\begin{array}{cc}
\left\{(x, y): x^{2}+(y-1)^{2}=k^{-1}\right\}, & \text { if } n-\left[\sqrt{\lambda_{n}}\right]+1 \leq k \leq n, k \\
\{(0,0)\}, & \text { is square integer } \\
\text { otherwise }
\end{array} .\right.
$$

Then the sequence $\left(A_{k}\right)$ is Wijsman $\lambda$-statistical convergent to $A=\{(0,0)\}$ since

$$
\lim _{n} \frac{1}{\lambda_{n}}\left|\left\{k \in I_{n}:\left|d\left(x, A_{k}\right)-d(x,\{(0,0)\})\right| \geq \varepsilon\right\}\right|=0 .
$$

But it is not Wijsman convergent.

Definition 2. Let $(X, \rho)$ be a metric space. Then for any non-empty closed subsets $A, A_{k} \subset X(k \in \mathbb{N})$, we say that the sequence $\left(A_{k}\right)$ is Wijsman $\lambda$-Cesáro summable to $A$ if

$$
\lim _{n} \frac{1}{\lambda_{n}} \sum_{k \in I_{n}}\left|d\left(x, A_{k}\right)-d(x, A)\right|=0 .
$$

In this case, we write $w_{\lambda}^{W}-\lim _{k} A_{k}=A$ or $A_{k} \rightarrow A\left(w_{\lambda}^{W}\right)$ and

$$
w_{\lambda}^{W}=\left\{\left(A_{k}\right): \lim _{n} \frac{1}{\lambda_{n}} \sum_{k \in I_{n}}\left|d\left(x, A_{k}\right)-d(x, A)\right|=0\right\} .
$$

If $\lambda_{n}=n$, then Wijsman $\lambda$-Cesáro summable becomes Wijsman-Cesáro summable, i.e.

$$
w^{W}=\left\{\left(A_{k}\right): \lim _{n} \frac{1}{n} \sum_{k \in \mathbb{N}}\left|d\left(x, A_{k}\right)-d(x, A)\right|=0\right\} .
$$


Theorem 1. Let $(X, \rho)$ be a metric space and $A, A_{k} \subset X(k \in \mathbb{N})$ be non-empty closed subsets of $X$. Then

(a) $w_{\lambda}^{W} \subset S_{\lambda}^{W}$ and the inclusion is proper.

(b) Let $\left(A_{k}\right) \in L_{\infty}$, then $S_{\lambda}^{W} \subset w_{\lambda}^{W}$.

(c) $S_{\lambda}^{W} \cap L_{\infty}=w_{\lambda}^{W} \cap L_{\infty}$.

Proof. (a) Let $\varepsilon>0$ and $\left(A_{k}\right) \in w_{\lambda}^{W}$. Then we can write

$$
\begin{aligned}
\sum_{k \in I_{n}}\left|d\left(x, A_{k}\right)-d(x, A)\right| & \sum_{\substack{k \in I_{n} \\
\left|d\left(x, A_{k}\right)-d(x, A)\right| \geq \varepsilon}}\left|d\left(x, A_{k}\right)-d(x, A)\right| \\
& \geq \varepsilon\left|\left\{k \in I_{n}:\left|d\left(x, A_{k}\right)-d(x, A)\right| \geq \varepsilon\right\}\right|
\end{aligned}
$$

which gives the result. To show that the inclusion is strict, we define the sequence $\left(A_{k}\right)$ as follows:

$$
A_{k}=\left\{\begin{array}{c}
\{k\}, \text { if } n-\left[\sqrt{\lambda_{n}}\right]+1 \leq k \leq n \\
\{0\}, \quad \text { otherwise }
\end{array}\right.
$$

It is clear that $\left(A_{k}\right) \notin L_{\infty}$ and for $\varepsilon>0$,

$$
\lim _{n} \frac{1}{\lambda_{n}}\left|\left\{k \in I_{n}:\left|d\left(x, A_{k}\right)-d(x,\{0\})\right| \geq \varepsilon\right\}\right|=\lim _{n} \frac{1}{\lambda_{n}}\left[\sqrt{\lambda_{n}}\right]=0 .
$$

So $\left(A_{k}\right) \in S_{\lambda}^{W}$, but

$$
\lim _{n} \frac{1}{\lambda_{n}} \sum_{k \in I_{n}}\left|d\left(x, A_{k}\right)-d(x,\{0\})\right|=\lim _{n} \frac{1}{\lambda_{n}} \frac{\left(\left[\sqrt{\lambda_{n}}\right]\left(\left[\sqrt{\lambda_{n}}\right]+1\right)\right)}{2}=\frac{1}{2} \neq 0 .
$$

Therefore $\left(A_{k}\right) \notin w_{\lambda}^{W}$. This completes the proof of (a).

(b) Suppose that $\left(A_{k}\right) \in S_{\lambda}^{W}$ and $\left(A_{k}\right) \in L_{\infty}$, say $\left|d\left(x, A_{k}\right)-d(x, A)\right| \leq M$ for each $x \in X$ and for all $k \in \mathbb{N}$. Given $\varepsilon>0$, we get

$$
\begin{aligned}
& \frac{1}{\lambda_{n}} \sum_{k \in I_{n}}\left|d\left(x, A_{k}\right)-d(x, A)\right| \\
& =\frac{1}{\lambda_{n}} \sum_{\substack{k \in I_{n} \\
\left|d\left(x, A_{k}\right)-d(x, A)\right| \geq \varepsilon}}\left|d\left(x, A_{k}\right)-d(x, A)\right| \\
& \quad+\frac{1}{\lambda_{n}} \sum_{\substack{k \in I_{n} \\
\left|d\left(x, A_{k}\right)-d(x, A)\right|<\varepsilon}}\left|d\left(x, A_{k}\right)-d(x, A)\right| \\
& \leq \frac{M}{\lambda_{n}}\left|\left\{k \in I_{n}:\left|d\left(x, A_{k}\right)-d(x, A)\right| \geq \varepsilon\right\}\right|+\varepsilon
\end{aligned}
$$

from which the result follows. 
(c) It follows from (a) and (b).

If we let $\lambda_{n}=n$ in Theorem 1, then we have the following corollary.

Corollary 1. Let $(X, \rho)$ be a metric space and $A, A_{k} \subset X(k \in \mathbb{N})$ be non-empty closed subsets of $X$. Then

(a) $w^{W} \subset S^{W}$ and the inclusion is proper.

(b) Let $\left(A_{k}\right) \in L_{\infty}$, then $S^{W} \subset w^{W}$.

(c) $S^{W} \cap L_{\infty}=w^{W} \cap L_{\infty}$.

Theorem 2. $S^{W} \subset S_{\lambda}^{W}$ if and only if $\liminf \frac{\lambda_{n}}{n}>0$.

Proof. Suppose that liminf $\frac{\lambda_{n}}{n}>0$. For given $\varepsilon>0$, we have

$\left\{k \leq n:\left|d\left(x, A_{k}\right)-d(x, A)\right| \geq \varepsilon\right\} \supset\left\{k \in I_{n}:\left|d\left(x, A_{k}\right)-d(x, A)\right| \geq \varepsilon\right\}$.

Therefore

$$
\begin{gathered}
\frac{1}{n}\left|\left\{k \leq n:\left|d\left(x, A_{k}\right)-d(x, A)\right| \geq \varepsilon\right\}\right| \geq \frac{1}{n}\left|\left\{k \in I_{n}:\left|d\left(x, A_{k}\right)-d(x, A)\right| \geq \varepsilon\right\}\right| \\
\geq \frac{\lambda_{n}}{n} \cdot \frac{1}{\lambda_{n}}\left|\left\{k \in I_{n}:\left|d\left(x, A_{k}\right)-d(x, A)\right| \geq \varepsilon\right\}\right| .
\end{gathered}
$$

Taking the limit as $n \rightarrow \infty$ and using $\liminf \frac{\lambda_{n}}{n}>0$, we get the desired result.

Conversely, suppose that $\liminf _{n} \frac{\lambda_{n}}{n}=0$. Then we can select a subsequence $(n(i))_{i=1}^{\infty}$ such that

We define a sequence $\left(A_{k}\right)$ as follows:

$$
\frac{\lambda_{n(i)}}{n(i)}<\frac{1}{i}
$$

$$
A_{k}=\left\{\begin{array}{lc}
\{1\}, & \text { if } n(i)-\left[\sqrt{\lambda_{n(i)}}\right]+1 \leq k \leq n(i), i=1,2,3, \ldots ; \\
\{0\}, & \text { otherwise. }
\end{array}\right.
$$

Then $\left(A_{k}\right)$ is Wijsman-statistically convergent, so $\left(A_{k}\right) \in S^{W}$. But $\left(A_{k}\right) \notin w_{\lambda}^{W}$. Therefore Theorem 1 (b) implies that $\left(A_{k}\right) \notin S_{\lambda}^{W}$. This completes the proof.

Theorem 3. $S_{\lambda}^{W} \subset s^{W}$ if $\liminf \frac{\lambda_{n}}{n}=1$.

Proof. Since $\lim _{n} \frac{\lambda_{n}}{n}=1$, then for $\varepsilon>0$, we observe that

$$
\begin{aligned}
& \frac{1}{n}\left|\left\{k \leq n:\left|d\left(x, A_{k}\right)-d(x, A)\right| \geq \varepsilon\right\}\right| \\
& \leq \frac{1}{n}\left|\left\{k \leq n-\lambda_{n}:\left|d\left(x, A_{k}\right)-d(x, A)\right| \geq \varepsilon\right\}\right| \\
& \quad+\frac{1}{n}\left|\left\{k \in I_{n}:\left|d\left(x, A_{k}\right)-d(x, A)\right| \geq \varepsilon\right\}\right| \\
& \leq \frac{n-\lambda_{n}}{n}+\frac{1}{n} \mid\left\{k \in I_{n}:\left|d\left(x, A_{k}-d(x, A) \mid \geq \varepsilon\right\}\right|\right.
\end{aligned}
$$




$$
=\frac{n-\lambda_{n}}{n}+\frac{\lambda_{n}}{n} \frac{1}{\lambda_{n}} \mid\left\{k \in I_{n}:\left|d\left(x, A_{k}-d(x, A) \mid \geq \varepsilon\right\}\right| .\right.
$$

This implies that $\left(A_{k}\right)$ Wijsman-statistically convergent, if $\left(A_{k}\right)$ is Wijsman $\lambda$ statistically convergent. Thus $S_{\lambda}^{W} \subset S^{W}$.

Remark 1. Since $\lim _{n} \frac{\lambda_{n}}{n}=1$, this implies that $\liminf _{n} \frac{\lambda_{n}}{n}>0$, then from Theorem 3, we have $S^{W} \subset S_{\lambda}^{W}$. Hence $S_{\lambda}^{W}=S^{W}$.

\section{WIJSMAN $\lambda$-STATISTICAL CONVERGENCE OF ORDER $\alpha$}

The notion of order statistical convergence was introduced by Gadjiev and Orhan [10] and after that statistical convergence of order $\alpha$ studied by Çolak [3], $\lambda$-statistical convergence of order $\alpha$ studied by Çolak and Bektaş [4], $\lambda$-statistical convergence of order $\alpha$ of sequence of functions studied by Et et al., [6,7] and many authors.

In this section, we define the concept of $S_{\lambda, \alpha}^{W}$-statistical convergence and establish the relationship of $S_{\lambda}^{W}$ with $w_{\lambda}^{W}$. Also we introduce the notion of $S_{\lambda}^{W}$-statistical convergence of order $\alpha$ of real number sequences and obtain some inclusion relations between the set of $S^{W}$-statistical convergence of order $\alpha$.

Definition 3. Let $(X, \rho)$ be a metric space, $\alpha \in(0,1]$ and $\lambda=\left(\lambda_{n}\right)$ be a nondecreasing sequence of positive numbers such that $\lambda_{n+1} \leq \lambda_{n}, \lambda_{1}=1, \lambda_{n} \rightarrow \infty$ as $n \rightarrow \infty$ and $I_{n}=\left[n-\lambda_{n}+1, n\right]$. We denote the family of such sequences $\lambda=\left(\lambda_{n}\right)$ by $\Lambda$. For any non-empty closed subsets $A, A_{k} \subset X(k \in \mathbb{N})$, we say that the sequence $\left(A_{k}\right)$ is Wijsman $\lambda$-statistical convergent to A of order $\alpha$ or $S_{\lambda, \alpha}^{W}$-convergent to $A$, if for every $\varepsilon>0$ and for each $x \in X$

$$
\lim _{n} \frac{1}{\lambda_{n}^{\alpha}}\left|\left\{k \in I_{n}:\left|d\left(x, A_{k}\right)-d(x, A)\right| \geq \varepsilon\right\}\right|=0 .
$$

In this case we write $S_{\lambda, \alpha}^{W}-\lim A_{k}=A$ or $A_{k} \rightarrow A\left(S_{\lambda, \alpha}^{W}\right)$. If $\lambda_{n}=n$ for all $n \in \mathbb{N}$, we obtain the sequence space $S_{\alpha}^{W}$ such that

$$
S_{\alpha}^{W}=\left\{\left(A_{k}\right): \lim _{n} \frac{1}{n^{\alpha}}\left|\left\{k \in I_{n}:\left|d\left(x, A_{k}\right)-d(x, A)\right| \geq \varepsilon\right\}\right|=0\right\}
$$

and if $\lambda_{n}=n$ for all $n \in \mathbb{N}$ and $\alpha=1$ we obtain the sequence space $S^{W}$ such that

$$
S^{W}=\left\{\left(A_{k}\right): \lim _{n} \frac{1}{n}\left|\left\{k \in I_{n}:\left|d\left(x, A_{k}\right)-d(x, A)\right| \geq \varepsilon\right\}\right|=0\right\} .
$$

Definition 4. Let $(X, \rho)$ be a metric space, $\alpha \in(0,1]$ and $\lambda=\left(\lambda_{n}\right) \in \Lambda$. For any non-empty closed subsets $A, A_{k} \subset X(k \in \mathbb{N})$, we say that the sequence $\left(A_{k}\right)$ is Wijsman $\lambda$-Cesaro summable to A of order $\alpha$ or $w_{\lambda, \alpha}^{W}$-convergent to $A$, if for each $x \in X$

$$
\lim _{n} \frac{1}{\lambda_{n}^{\alpha}} \sum_{k \in I_{n}}\left|d\left(x, A_{k}\right)-d(x, A)\right|=0 .
$$


In this case we write $w_{\lambda, \alpha}^{W}-\lim A_{k}=A$ or $A_{k} \rightarrow A\left(w_{\lambda, \alpha}^{W}\right)$. If $\lambda_{n}=n$ for all $n \in \mathbb{N}$, we obtain the sequence space $w_{\alpha}^{W}$ such that

$$
w_{\alpha}^{W}=\left\{\left(A_{k}\right): \lim _{n} \frac{1}{n^{\alpha}} \sum_{k \in I_{n}}\left|d\left(x, A_{k}\right)-d(x, A)\right|=0\right\}
$$

and if $\lambda_{n}=n$ for all $n \in \mathbb{N}$ and $\alpha=1$ we obtain the sequence space $w^{W}$ such that

$$
w^{W}=\left\{\left(A_{k}\right): \lim _{n} \frac{1}{n} \sum_{k}\left|d\left(x, A_{k}\right)-d(x, A)\right|=0=0\right\} .
$$

Theorem 4. Let $(X, \rho)$ be a metric space and $\alpha \in(0,1]$. If $S_{\lambda, \alpha}^{W}-\lim A_{k}=A$ then $A$ is unique.

Proof. The proof is easy, so omitted.

Theorem 5. Let $(X, \rho)$ be a metric space, $\alpha \in(0,1]$ and non-empty closed subsets $A, B, A_{k}, B_{k} \subset X(k \in \mathbb{N})$, then

(a) If $S_{\lambda, \alpha}^{W}-\lim A_{k}=A$ and $c \in \mathbb{C}$, then $S_{\lambda, \alpha}^{W}-\lim c A_{k}=c A$;

(b) If $S_{\lambda, \alpha}^{W}-\lim A_{k}=A$ and $S_{\lambda, \alpha}^{W}-\lim B_{k}=B$, then $S_{\lambda, \alpha}^{W}-\lim \left(A_{k}+B_{k}\right)=$ $A+B$.

Proof. (a) For $c=0$, the result is trivial. Suppose that $c \neq 0$, then for every $\varepsilon>0$ the result follows form the following inequality

$$
\begin{aligned}
\frac{1}{\lambda_{n}^{\alpha}} \mid\left\{k \in I_{n}: \mid d\left(c x, c A_{k}\right)-d\right. & (c x, c A) \mid \geq \varepsilon\} \mid \\
= & \frac{1}{\lambda_{n}^{\alpha}}\left|\left\{k \in I_{n}:\left|d\left(x, A_{k}\right)-d(x, A)\right| \geq \frac{\varepsilon}{|c|}\right\}\right| .
\end{aligned}
$$

(b) For every $\varepsilon>0$. The result follows from the following inequality.

$$
\begin{aligned}
& \frac{1}{\lambda_{n}^{\alpha}}\left|\left\{k \in I_{n}:\left|d\left(x, A_{k}+B_{k}\right)-d(x, A+B)\right| \geq \varepsilon\right\}\right| \\
& \leq \frac{1}{\lambda_{n}^{\alpha}}\left|\left\{k \in I_{n}:\left|d\left(x, A_{k}\right)-d(x, A)\right| \geq \frac{\varepsilon}{2}\right\}\right| \\
& \quad+\frac{1}{\lambda_{n}^{\alpha}}\left|\left\{k \in I_{n}:\left|d\left(x, B_{k}\right)-d(x, B)\right| \geq \frac{\varepsilon}{2}\right\}\right| .
\end{aligned}
$$

If we take $\lambda_{n}=n$ in the above theorem, then we have

Corollary 2. Let $(X, \rho)$ be a metric space, $\alpha \in(0,1]$ and non-empty closed subsets $A, B, A_{k}, B_{k} \subset X(k \in \mathbb{N})$, then 
(a) If $S_{\alpha}^{W}-\lim A_{k}=A$ and $c \in \mathbb{C}$, then $S_{\alpha}^{W}-\lim c A_{k}=c A$;

(b) If $S_{\alpha}^{W}-\lim A_{k}=A$ and $S_{\alpha}^{W}-\lim B_{k}=B$, then $S_{\alpha}^{W}-\lim \left(A_{k}+B_{k}\right)=$ $A+B$.

Theorem 6. If $0<\alpha<\beta \leq 1$, then $S_{\lambda, \alpha}^{W} \subset S_{\lambda, \beta}^{W}$ and the inclusion is strict.

Proof. The proof of the result follows form the following inequality.

$$
\begin{aligned}
\frac{1}{\lambda_{n}^{\beta}} \mid\left\{k \in I_{n}:\left|d\left(x, A_{k}\right)-d(x, A)\right|\right. & \geq \varepsilon\} \mid \\
& \geq \frac{1}{\lambda_{n}^{\alpha}}\left|\left\{k \in I_{n}:\left|d\left(x, A_{k}\right)-d(x, A)\right| \geq \varepsilon\right\}\right| .
\end{aligned}
$$

To prove the inclusion is strict, let $\lambda$ be given and we consider a sequence $\left(A_{k}\right)$ of non-empty closed subsets of $(\mathrm{X}, \rho)$ metric space defined by

$$
\begin{aligned}
A_{k} & =\left\{\begin{array}{lc}
\{(k, k)\}, & \text { if } n-\left[\sqrt{\lambda_{n}}\right]+1 \leq k \leq n ; \\
\{(0,0)\}, & \text { otherwise. }
\end{array}\right. \\
& =\frac{1}{\lambda_{n}^{\beta}}\left|\left\{k \in I_{n}: n-\left[\sqrt{\lambda_{n}}\right]+1 \leq k \leq n\right\}\right| \leq \frac{\sqrt{\lambda_{n}}}{\lambda_{n}^{\beta}}
\end{aligned}
$$

Then we have $\left(A_{k}\right) \in S_{\lambda, \beta}^{W}$ for $\frac{1}{2}<\beta \leq 1$ but $\left(A_{k}\right) \notin S_{\lambda, \alpha}^{W}$ for $0<\alpha \leq \frac{1}{2}$.

Theorem 7. If a sequence $\left(A_{k}\right)$ of non-empty closed subsets of $(X, \rho)$ metric space is $S_{\lambda, \alpha}^{W}$-convergent to $A$, then it is $S_{\lambda}^{W}$-convergent to $A$ for $0<\alpha \leq 1$.

Proof. It is easy, so omitted.

Theorem 8. Let $(X, \rho)$ be a metric space and $\alpha \in(0,1]$. Then $S_{\alpha}^{W} \subset S_{\lambda, \alpha}^{W}$ if

$$
\lim _{n \rightarrow \infty} \inf \frac{\lambda_{n}^{\alpha}}{n^{\alpha}}>0
$$

Proof. If $A_{k} \rightarrow A\left(S_{\alpha}^{W}\right)$ then for every $\varepsilon>0$ and for sufficiently large $n$ we have

$$
\begin{aligned}
\frac{1}{n^{\alpha}} \mid\left\{k \leq n:\left|d\left(x, A_{k}\right)-d(x, A)\right|\right. & \geq \varepsilon\} \mid \\
\geq \frac{1}{n^{\alpha}} \mid\left\{k \in I_{n}:\right. & \left.\left|d\left(x, A_{k}\right)-d(x, A)\right| \geq \varepsilon\right\} \mid \\
& \geq \frac{\lambda_{n}^{\alpha}}{n^{\alpha}} \frac{1}{\lambda_{n}^{\alpha}}\left|\left\{k \in I_{n}:\left|d\left(x, A_{k}\right)-d(x, A)\right| \geq \varepsilon\right\}\right| .
\end{aligned}
$$

Taking the limit as $n \rightarrow \infty$ and using the given condition, we get $A_{k} \rightarrow A\left(S_{\lambda, \alpha}^{W}\right)$. This completes the proof of the theorem.

Corollary 3. Let $(X, \rho)$ be a metric space, $\lambda=\left(\lambda_{n}\right) \in \Lambda$ and $\alpha \in(0,1]$. Then $S_{\lambda, \alpha}^{W} \subset S^{W}$. 
Theorem 9. Let $\lambda=\left(\lambda_{n}\right) \in \Lambda$ and $\alpha \in(0,1]$. Then $S^{W} \subset S_{\lambda, \alpha}^{W}$ if and only if

$$
\lim _{n \rightarrow \infty} \inf \frac{\lambda_{n}^{\alpha}}{n}>0
$$

Proof. Let the condition (3.1) holds and $\left(A_{k}\right) \in S^{W}$. For a given $\varepsilon>0$ we have

$$
\left\{k \leq n:\left|d\left(x, A_{k}\right)-d(x, A)\right| \geq \varepsilon\right\} \supset\left\{k \in I_{n}:\left|d\left(x, A_{k}\right)-d(x, A)\right| \geq \varepsilon\right\} .
$$

Then we have

$$
\begin{aligned}
\frac{1}{n} \mid\left\{k \leq n:\left|d\left(x, A_{k}\right)-d(x, A)\right|\right. & \geq \varepsilon\}\left|\geq \frac{1}{n}\right|\left\{k \in I_{n}:\left|d\left(x, A_{k}\right)-d(x, A)\right| \geq \varepsilon\right\} \mid \\
= & \frac{\lambda_{n}^{\alpha}}{n} \frac{1}{\lambda_{n}^{\alpha}}\left|\left\{k \in I_{n}:\left|d\left(x, A_{k}\right)-d(x, A)\right| \geq \varepsilon\right\}\right| .
\end{aligned}
$$

By taking limit as $n \rightarrow \infty$ and from relation (3.1) we have

$$
A_{k} \rightarrow A\left(S^{W}\right) \Rightarrow A_{k} \rightarrow A\left(S_{\lambda, \alpha}^{W}\right) \text {. }
$$

Next we suppose that

$$
\lim _{n \rightarrow \infty} \inf \frac{\lambda_{n}^{\alpha}}{n}=0
$$

Then we can choose a subsequence $\left(n_{i}\right)$ such that $\frac{\lambda_{n_{i}}^{\alpha}}{n_{i}}<\frac{1}{i}$. Define a sequence $\left(A_{k}\right)$ as follows:

$$
A_{k}= \begin{cases}\{(1,1)\}, & \text { if } k \in I_{n_{i}} \\ \{(0,0)\}, & \text { otherwise. }\end{cases}
$$

Then clearly $\left(A_{k}\right) \in S^{W}$ but $\left(A_{k}\right) \notin S_{\lambda, \alpha}^{W}$. Since $S_{\lambda, \alpha}^{W} \subset S_{\lambda}^{W}$, we have $\left(A_{k}\right) \notin S_{\lambda, \alpha}^{W}$, which is a contradiction. Hence the relation (3.1) holds.

Theorem 10. Let $(X, \rho)$ be a metric space, $\lambda=\left(\lambda_{n}\right)$ and $\mu=\left(\mu_{n}\right)$ be two sequences in $\Lambda$ such that $\lambda_{n} \leq \mu_{n}$ for all $n \in \mathbb{N}$ and $0<\alpha \leq \beta \leq 1$. If

$$
\lim _{n \rightarrow \infty} \inf \frac{\lambda_{n}^{\alpha}}{\mu_{n}^{\beta}}
$$

then $S_{\lambda, \beta}^{W} \subseteq S_{\lambda, \alpha}^{W}$

Proof. Suppose that $\lambda_{n} \leq \mu_{n}$ for all $n \in \mathbb{N}$ and the condition (3.2) satisfied. Then $I_{n} \subset J_{n}$ and so that for $\varepsilon>0$ we can write

$$
\left\{k \in J_{n}:\left|d\left(x, A_{k}\right)-d(x, A)\right| \geq \varepsilon\right\} \supset\left\{k \in I_{n}:\left|d\left(x, A_{k}\right)-d(x, A)\right| \geq \varepsilon\right\} .
$$

Then we have

$$
\frac{1}{\mu_{n}^{\beta}}\left|\left\{k \in J_{n}:\left|d\left(x, A_{k}\right)-d(x, A)\right| \geq \varepsilon\right\}\right|
$$




$$
\geq \frac{\lambda_{n}^{\alpha}}{\mu_{n}^{\beta}} \frac{1}{\lambda_{n}^{\alpha}}\left|\left\{k \in I_{n}:\left|d\left(x, A_{k}\right)-d(x, A)\right| \geq \varepsilon\right\}\right|,
$$

for all $n \in \mathbb{N}$, where $J_{n}=\left[n-\mu_{n}+1, n\right]$. Taking limit $n \rightarrow \infty$ in the last inequality and using (3.2), we have $S_{\lambda, \beta}^{W} \subseteq S_{\lambda, \alpha}^{W}$.

Corollary 4. Let $(X, \rho)$ be a metric space, $\lambda=\left(\lambda_{n}\right)$ and $\mu=\left(\mu_{n}\right)$ be two sequences in $\Lambda$ such that $\lambda_{n} \leq \mu_{n}$ for all $n \in \mathbb{N}$. If (3.2) holds, then
(a) $S_{\mu, \alpha}^{W} \subseteq S_{\lambda, \alpha}^{W}$ for $0<\alpha \leq 1$,
(b) $S_{\mu}^{W} \subseteq S_{\lambda, \alpha}^{W}$ for $0<\alpha \leq 1$,
(c) $S_{\mu}^{W} \subseteq S_{\lambda}^{W}$.

Theorem 11. Let $(X, \rho)$ be a metric space, $\lambda=\left(\lambda_{n}\right)$ and $\mu=\left(\mu_{n}\right)$ be two sequences in $\Lambda$ such that $\lambda_{n} \leq \mu_{n}$ for all $n \in \mathbb{N}$ and $0<\alpha \leq \beta \leq 1$. If

$$
\lim _{n \rightarrow \infty} \frac{\mu_{n}}{\lambda_{n}^{\beta}}=1,
$$

then $S_{\lambda, \alpha}^{W} \subseteq S_{\mu, \beta}^{W}$.

Proof. Let $S_{\lambda, \alpha}^{W}-\lim A_{k}=A$ and (3.3) be satisfied. Since $I_{n} \subset J_{n}$, for $\varepsilon>0$ we can write

$$
\begin{aligned}
& \frac{1}{\mu_{n}^{\beta}}\left|\left\{k \in J_{n}:\left|d\left(x, A_{k}\right)-d(x, A)\right| \geq \varepsilon\right\}\right| \\
& =\frac{1}{\mu_{n}^{\beta}}\left|\left\{n-\mu_{n}+1 \leq k \leq n-\lambda_{n}:\left|d\left(x, A_{k}\right)-d(x, A)\right| \geq \varepsilon\right\}\right| \\
& \quad+\frac{1}{\mu_{n}^{\beta}}\left|\left\{k \in I_{n}:\left|d\left(x, A_{k}\right)-d(x, A)\right| \geq \varepsilon\right\}\right| \\
& \leq \frac{\mu_{n}-\lambda_{n}}{\mu_{n}^{\beta}}+\frac{1}{\mu_{n}^{\beta}}\left|\left\{k \in I_{n}:\left|d\left(x, A_{k}\right)-d(x, A)\right| \geq \varepsilon\right\}\right| \\
& \leq \frac{\mu_{n}-\lambda_{n}^{\beta}}{\lambda_{n}^{\beta}}+\frac{1}{\mu_{n}^{\beta}}\left|\left\{k \in I_{n}:\left|d\left(x, A_{k}\right)-d(x, A)\right| \geq \varepsilon\right\}\right| \\
& \leq\left(\frac{\mu_{n}}{\lambda_{n}^{\beta}}-1\right)+\frac{\lambda_{n}^{\alpha}}{\mu_{n}^{\beta}} \frac{1}{\lambda_{n}^{\alpha}}\left|\left\{k \in I_{n}:\left|d\left(x, A_{k}\right)-d(x, A)\right| \geq \varepsilon\right\}\right| .
\end{aligned}
$$

Using the relation (3.3) and $S_{\lambda, \alpha}^{W}-\lim A_{k}=A$ the right-hand side of the above inequality tends to zero as $n \rightarrow \infty$. This implies that $S_{\lambda, \alpha}^{W} \subseteq S_{\mu, \beta}^{W}$.

Corollary 5. Let $(X, \rho)$ be a metric space, $\lambda=\left(\lambda_{n}\right)$ and $\mu=\left(\mu_{n}\right)$ be two sequences in $\Lambda$ such that $\lambda_{n} \leq \mu_{n}$ for all $n \in \mathbb{N}$. If (3.3) holds, then

(a) $S_{\lambda, \alpha}^{W} \subseteq S_{\mu, \alpha}^{W}$ for $0<\alpha \leq 1$, 
(b) $S_{\lambda}^{W} \subseteq S_{\mu, \alpha}^{W}$ for $0<\alpha \leq 1$,

(c) $S_{\lambda}^{W} \subseteq S_{\mu}^{W}$.

An Orlicz function $M$ is a function $M:[0, \infty) \rightarrow[0, \infty)$ which is continuous, convex, nondecreasing function define for $x>0$ such that $M(0)=0, M(x)>0$ and $M(x) \rightarrow \infty$ as $x \rightarrow \infty$. If convexity of Orlicz function is replaced by $M(x+y) \leq$ $M(x)+M(y)$ then this function is called the modulus function and characterized by Ruckle [19]. An Orlicz function $M$ is said to satisfy $\Delta_{2}-$ condition for all values u, if there exists $K>0$ such that $M(2 u) \leq K M(u), u \geq 0$.

Lemma 1. An Orlicz function satisfies the inequality $M(\lambda x) \leq \lambda M(x)$ for all $\lambda$ with $0<\lambda<1$.

Lindenstrauss and Tzafriri [16] used the idea of Orlicz function to construct the sequence space

$$
l_{M}=\left\{\left(x_{k}\right): \sum_{k=1}^{\infty} M\left(\frac{\left|x_{k}\right|}{r}\right)<\infty, \text { for some } r>0\right\},
$$

which is a Banach space normed by

$$
\left\|\left(x_{k}\right)\right\|=\inf \left\{r>0: \sum_{k=1}^{\infty} M\left(\frac{\left|x_{k}\right|}{r}\right) \leq 1\right\} .
$$

The space $l_{M}$ is closely related to the space $l_{p}$, which is an Orlicz sequence space with $M(x)=|x|^{p}$, for $1 \leq p<\infty$.

Definition 5. Let $(X, \rho)$ be a metric space. Let $M$ be an Orlicz function, $p=\left(p_{k}\right)$ be a sequence of strictly positive real numbers, $\alpha \in(0,1], \lambda=\left(\lambda_{n}\right)$ be a sequence of positive reals, and for $\rho>0$, now we define

$$
\begin{gathered}
w_{\lambda, \alpha}^{W}[M, p]=\left\{\left(A_{k}\right) \in X: \lim _{n \rightarrow \infty} \frac{1}{\lambda_{n}^{\alpha}} \sum_{k \in I_{n}}\left[M\left(\frac{\left|d\left(x, A_{k}\right)-d(x, A)\right|}{\rho}\right)\right]^{p_{k}}=0,\right. \\
\text { for some } A \text { and for } x \in X\} .
\end{gathered}
$$

If $M(x)=x$ and $p_{k}=p$ for all $k \in \mathbb{N}$ then we shall write $w_{\lambda, \alpha}^{W}[M, p]=w_{\lambda, \alpha}^{W}(p)$ and if $M(x)=x$ then we shall write $w_{\lambda, \alpha}^{W}[M, p]=w_{\lambda, \alpha}^{W}[p]$.

Theorem 12. Let $\left(p_{k}\right)$ be a bounded and $0<\inf _{k} p_{k} \leq p_{k} \leq \sup _{k} p_{k}=H<\infty$. Let $0<\alpha \leq \beta \leq 1, M$ be an Orlicz function and $\lambda=\left(\lambda_{n}\right)$ be a sequence of positive reals, then $w_{\lambda, \alpha}^{W}[M, p] \subset S_{\lambda, \beta}^{W}$. 
Proof. Let $\left(A_{k}\right) \in w_{\lambda, \alpha}^{W}[M, p]$. Let $\varepsilon>0$ be given. As $\lambda_{n}^{\alpha} \leq \lambda_{n}^{\beta}$ for each $n$ we can write

$$
\begin{aligned}
& \frac{1}{\lambda_{n}^{\alpha}} \sum_{k \in I_{n}}\left[M\left(\frac{\left|d\left(x, A_{k}\right)-d(x, A)\right|}{\rho}\right)\right]^{p_{k}} \\
& =\frac{1}{\lambda_{n}^{\alpha}}\left[\sum_{\substack{k \in I_{n} \\
\left|d\left(x, A_{k}\right)-d(x, A)\right| \geq \varepsilon}}\left[M\left(\frac{\left|d\left(x, A_{k}\right)-d(x, A)\right|}{\rho}\right)\right]^{p_{k}}\right. \\
& \left.+\sum_{\substack{k \in I_{n} \\
\left|d\left(x, A_{k}\right)-d(x, A)\right|<\varepsilon}}\left[M\left(\frac{\left|d\left(x, A_{k}\right)-d(x, A)\right|}{\rho}\right)\right]^{p_{k}}\right] \\
& \geq \frac{1}{\lambda_{n}^{\beta}}\left[\sum_{\substack{k \in I_{n} \\
\left|d\left(x, A_{k}\right)-d(x, A)\right| \geq \varepsilon}}\left[M\left(\frac{\left|d\left(x, A_{k}\right)-d(x, A)\right|}{\rho}\right)\right]^{p_{k}}\right. \\
& \left.+\sum_{\substack{k \in I_{n} \\
\left|d\left(x, A_{k}\right)-d(x, A)\right|<\varepsilon}}\left[M\left(\frac{\left|d\left(x, A_{k}\right)-d(x, A)\right|}{\rho}\right)\right]^{p_{k}}\right] \\
& \geq \frac{1}{\lambda_{n}^{\beta}} \sum_{\substack{k \in I_{n} \\
\left|d\left(x, A_{k}\right)-d(x, A)\right| \geq \varepsilon}}\left[M\left(\frac{\varepsilon}{\rho}\right)\right]^{p_{k}} \\
& \geq \frac{1}{\lambda_{n}^{\beta}} \sum_{\substack{k \in I_{n} \\
\left|d\left(x, A_{k}\right)-d(x, A)\right| \geq \varepsilon}} \min \left(\left[M\left(\varepsilon_{1}\right)\right]^{h},\left[M\left(\varepsilon_{1}\right)\right]^{H}\right) \\
& \geq \frac{1}{\lambda_{n}^{\beta}}\left|\left\{k \in I_{n}:\left|d\left(x, A_{k}\right)-d(x, A)\right| \geq \varepsilon\right\}\right| \min \left(\left[M\left(\varepsilon_{1}\right)\right]^{h},\left[M\left(\varepsilon_{1}\right)\right]^{H}\right),
\end{aligned}
$$

where $\varepsilon_{1}=\frac{\varepsilon}{\rho}$. From the above inequality we have $\left(A_{k}\right) \in S_{\lambda, \alpha}^{W}$.

Corollary 6. Let $0<\alpha \leq 1, M$ be an Orlicz function and $\lambda=\left(\lambda_{n}\right)$ be an element of $\Lambda$, then $w_{\lambda, \alpha}^{W}[M, p] \subset S_{\lambda, \alpha}^{W}$.

Theorem 13. Let $M$ be an Orlicz function, $\left(A_{k}\right)$ be a sequence in $L_{\infty}$, and $\lambda=$ $\left(\lambda_{n}\right)$ be an element of $\Lambda$. If $\lim _{n \rightarrow \infty} \frac{\lambda_{n}}{\lambda_{n}^{\alpha}}=1$, then $S_{\lambda, \alpha}^{W} \subset w_{\lambda, \alpha}^{W}[M, p]$. 
Proof. Suppose that $\left(A_{k}\right)$ is in $L_{\infty}$ and $S_{\lambda, \alpha}^{W}-\lim _{k} A_{k}=A$. As $\left(A_{k}\right) \in L_{\infty}$ there exists $K>0$ such that $\left|d\left(x, A_{k}\right)-d(x, A)\right| \leq K$ for all $k$. For given $\varepsilon>0$ we have

$$
\begin{aligned}
& \frac{1}{\lambda_{n}^{\alpha}} \sum_{k \in I_{n}}\left[M\left(\frac{\left|d\left(x, A_{k}\right)-d(x, A)\right|}{\rho}\right)\right]^{p_{k}} \\
& =\frac{1}{\lambda_{n}^{\alpha}} \sum_{\substack{k \in I_{n} \\
\left|d\left(x, A_{k}\right)-d(x, A)\right| \geq \varepsilon}}\left[M\left(\frac{\left|d\left(x, A_{k}\right)-d(x, A)\right|}{\rho}\right)\right]^{p_{k}} \\
& +\frac{1}{\lambda_{n}^{\alpha}} \sum_{\substack{k \in I_{n} \\
\left|d\left(x, A_{k}\right)-d(x, A)\right|<\varepsilon}}\left[M\left(\frac{\left|d\left(x, A_{k}\right)-d(x, A)\right|}{\rho}\right)\right]^{p_{k}} \\
& \leq \frac{1}{\lambda_{n}^{\alpha}} \sum_{\substack{k \in I_{n} \\
\left|d\left(x, A_{k}\right)-d(x, A)\right| \geq \varepsilon}} \max \left\{\left[M\left(\frac{K}{\rho}\right)\right]^{h},\left[M\left(\frac{K}{\rho}\right)\right]^{H}\right\} \\
& +\frac{1}{\lambda_{n}^{\alpha}} \sum_{\substack{k \in I_{n} \\
\left|d\left(x, A_{k}\right)-d(x, A)\right|<\varepsilon}}\left[M\left(\frac{\varepsilon}{\rho}\right)\right]^{p_{k}} \\
& \leq \max \left\{\left[M\left(\frac{K}{\rho}\right)\right]^{h},\left[M\left(\frac{K}{\rho}\right)\right]^{H}\right\} \frac{1}{\lambda_{n}^{\alpha}}\left|d\left(x, A_{k}\right)-d(x, A)\right| \geq \varepsilon \mid \\
& \quad+\frac{\lambda_{n}}{\lambda_{n}^{\alpha}} \max \left\{\left[M\left(\frac{\varepsilon}{\rho}\right)\right]^{h},\left[M\left(\frac{\varepsilon}{\rho}\right)\right]^{H}\right\} .
\end{aligned}
$$

Therefore we have $\left(A_{k}\right) \in w_{\lambda, \alpha}^{W}[M, p]$.

Theorem 14. Let $\lambda=\left(\lambda_{n}\right) \in \Lambda, 0<\alpha \leq \beta \leq 1$, $p$ be a positive real number, then $w_{\lambda, \alpha}^{W}(p) \subseteq w_{\beta, \alpha}^{W}(p)$.

Proof. The proof is easy, so omitted.

Corollary 7. Let $\lambda=\left(\lambda_{n}\right) \in \Lambda$ and $p$ be a positive real number, then $w_{\lambda, \alpha}^{W}(p) \subseteq$ $w_{\lambda}^{W}(p)$.

Theorem 15. Let $\lambda=\left(\lambda_{n}\right) \in \Lambda, 0<\alpha \leq \beta \leq 1$ and $p$ be a positive real number, then $w_{\lambda, \alpha}^{W}(p) \subseteq S_{\lambda, \beta}^{W}$.

Proof. Let $\left(A_{k}\right) \in w_{\lambda, \alpha}^{W}(p)$ and for $\varepsilon>0$ we have

$$
\sum_{k \in I_{n}}\left|d\left(x, A_{k}\right)-d(x, A)\right|^{p}=\sum_{\substack{k \in I_{n} \\\left|d\left(x, A_{k}\right)-d(x, A)\right| \geq \varepsilon}}\left|d\left(x, A_{k}\right)-d(x, A)\right|^{p}
$$




$$
\begin{gathered}
+\sum_{\substack{k \in I_{n} \\
\left|d\left(x, A_{k}\right)-d(x, A)\right|<\varepsilon}}\left|d\left(x, A_{k}\right)-d(x, A)\right|^{p} \\
\geq \sum_{\substack{k \in I_{n} \\
\left|d\left(x, A_{k}\right)-d(x, A)\right| \geq \varepsilon}}\left|d\left(x, A_{k}\right)-d(x, A)\right|^{p} \\
\geq\left|\left\{k \in I_{n}:\left|d\left(x, A_{k}\right)-d(x, A)\right| \geq \varepsilon\right\}\right| \cdot \varepsilon^{p} .
\end{gathered}
$$

Therefore we have

$$
\frac{1}{\lambda_{n}^{\alpha}} \sum_{k \in I_{n}}\left|d\left(x, A_{k}\right)-d(x, A)\right|^{p} \geq \frac{1}{\lambda_{n}^{\beta}}\left|\left\{k \in I_{n}:\left|d\left(x, A_{k}\right)-d(x, A)\right| \geq \varepsilon\right\}\right| . \varepsilon^{p} .
$$

The last inequality implies that $\left(A_{k}\right) \in S_{\lambda, \beta}^{W}$ if $\left(A_{k}\right) \in w_{\lambda, \alpha}^{W}(p)$. This compltes the proof of the theorem.

Theorem 16. Let $\lambda=\left(\lambda_{n}\right)$ and $\mu=\left(\mu_{n}\right)$ be two sequences in $\Lambda$ such that $\lambda_{n} \leq$ $\mu_{n}$ for all $n \in \mathbb{N}$ and $0<\alpha \leq \beta \leq 1$. If (3.3) holds, then $w_{\mu, \beta}^{W}(p) \subseteq w_{\lambda, \alpha}^{W}(p)$.

Proof. The proof is easy, so omitted.

Corollary 8. Let $\lambda=\left(\lambda_{n}\right)$ and $\mu=\left(\mu_{n}\right)$ be two sequences in $\Lambda$ such that $\lambda_{n} \leq \mu_{n}$ for all $n \in \mathbb{N}$. If (3.3) holds, then

(a) $w_{\mu, \alpha}^{W}(p) \subseteq w_{\lambda, \alpha}^{W}(p)$ for $0<\alpha \leq 1$,

(b) $\left.w_{\mu}^{W}(p)\right) \subseteq w_{\lambda}^{W}(p)$ for $0<\alpha \leq 1$,

(c) $w_{\mu}^{W}(p) \subseteq w_{\lambda}^{W}(p)$.

Theorem 17. Let $\lambda=\left(\lambda_{n}\right)$ and $\mu=\left(\mu_{n}\right)$ be two sequences in $\Lambda$ such that $\lambda_{n} \leq$ $\mu_{n}$ for all $n \in \mathbb{N}$ and $0<\alpha \leq \beta \leq 1$. If (3.3) holds, then $w_{\mu, \beta}^{W}(p) \subseteq S_{\lambda, \alpha}^{W}$.

Proof. Let $\left(A_{k}\right) \in w_{\mu, \beta}^{W}(p)$ and for $\varepsilon>0$. Then we have

$$
\begin{aligned}
\sum_{k \in I_{n}}\left|d\left(x, A_{k}\right)-d(x, A)\right|^{p}= & \sum_{\substack{k \in I_{n} \\
\left|d\left(x, A_{k}\right)-d(x, A)\right| \geq \varepsilon}}\left|d\left(x, A_{k}\right)-d(x, A)\right|^{p} \\
& +\sum_{\substack{k \in I_{n} \\
\left|d\left(x, A_{k}\right)-d(x, A)\right|<\varepsilon}}\left|d\left(x, A_{k}\right)-d(x, A)\right|^{p} \\
\geq & \sum_{\substack{k \in I_{n} \\
\left|d\left(x, A_{k}\right)-d(x, A)\right| \geq \varepsilon}}\left|d\left(x, A_{k}\right)-d(x, A)\right|^{p} \\
\geq & \left|\left\{k \in I_{n}:\left|d\left(x, A_{k}\right)-d(x, A)\right| \geq \varepsilon\right\}\right| \cdot \varepsilon^{p} .
\end{aligned}
$$


Therefore we have

$$
\frac{1}{\mu_{n}^{\beta}} \sum_{k \in I_{n}}\left|d\left(x, A_{k}\right)-d(x, A)\right|^{p} \geq \frac{\lambda_{n}^{\alpha}}{\mu_{n}^{\beta}} \frac{1}{\lambda_{n}^{\alpha}}\left|\left\{k \in I_{n}:\left|d\left(x, A_{k}\right)-d(x, A)\right| \geq \varepsilon\right\}\right| . \varepsilon^{p} .
$$

Since (3.3) holds and $\left(A_{k}\right) \in w_{\mu, \beta}^{W}(p)$. The last inequality implies that $\left(A_{k}\right) \in S_{\lambda, \alpha}^{W}$. This completes the proof of the theorem.

Corollary 9. Let $\lambda=\left(\lambda_{n}\right)$ and $\mu=\left(\mu_{n}\right)$ be two sequences in $\Lambda$ such that $\lambda_{n} \leq \mu_{n}$ for all $n \in \mathbb{N}$ and $0<\alpha \leq 1$. If (3.3) holds, then
(a) $w_{\mu, \alpha}^{W}(p) \subseteq S_{\lambda, \alpha}^{W}$,
(b) $w_{\mu}^{W}(p) \subseteq S_{\lambda, \alpha}^{W}$,
(c) $w_{\mu}^{W}(p) \subseteq S_{\lambda}^{W}$.

Theorem 18. Let $\lambda=\left(\lambda_{n}\right)$ and $\mu=\left(\mu_{n}\right)$ be two sequences in $\Lambda$ such that $\lambda_{n} \leq$ $\mu_{n}$ for all $n \in \mathbb{N}$ and $0<\alpha \leq \beta \leq 1$. If (3.3) holds, then $L_{\infty} \cap w_{\mu, \alpha}^{W}(p) \subseteq w_{\lambda, \beta}^{W}(p)$.

Proof. Let $\left(A_{k}\right) \in L_{\infty} \cap w_{\mu, \alpha}^{W}(p)$ and suppose that (3.3) holds. Since $\left(A_{k}\right) \in L_{\infty}$, there esists $K>0$ such that $\left|d\left(x, A_{k}\right)-d(x, A)\right| \leq K$ for all $k$. Since $\lambda_{n} \leq \mu_{n}$ and $I_{n} \subset J_{n}$ for all $n \in \mathbb{N}$ we can write

$$
\begin{aligned}
& \frac{1}{\mu_{n}^{\beta}} \sum_{k \in J_{n}}\left|d\left(x, A_{k}\right)-d(x, A)\right|^{p} \\
& =\frac{1}{\mu_{n}^{\beta}} \sum_{k \in J_{n}-I_{n}}\left|d\left(x, A_{k}\right)-d(x, A)\right|^{p}+\frac{1}{\mu_{n}^{\beta}} \sum_{k \in I_{n}}\left|d\left(x, A_{k}\right)-d(x, A)\right|^{p} \\
& \leq\left(\frac{\mu_{n}-\lambda_{n}}{\mu_{n}^{\beta}}\right) K^{p}+\frac{1}{\mu_{n}^{\beta}} \sum_{k \in I_{n}}\left|d\left(x, A_{k}\right)-d(x, A)\right|^{p} \\
& \leq\left(\frac{\mu_{n}-\lambda_{n}^{\beta}}{\mu_{n}^{\beta}}\right) K^{p}+\frac{1}{\mu_{n}^{\beta}} \sum_{k \in I_{n}}\left|d\left(x, A_{k}\right)-d(x, A)\right|^{p} \\
& \leq\left(\frac{\mu_{n}-\lambda_{n}^{\beta}}{\lambda_{n}^{\beta}}\right) K^{p}+\frac{\lambda_{n}^{\alpha}}{\mu_{n}^{\beta}} \frac{1}{\lambda_{n}^{\alpha}} \sum_{k \in I_{n}}\left|d\left(x, A_{k}\right)-d(x, A)\right|^{p} \\
& \leq\left(\frac{\mu_{n}}{\lambda_{n}^{\beta}-1}\right) K^{p}+\frac{\lambda_{n}^{\alpha}}{\mu_{n}^{\beta}} \frac{1}{\lambda_{n}^{\alpha}} \sum_{k \in I_{n}}\left|d\left(x, A_{k}\right)-d(x, A)\right|^{p} .
\end{aligned}
$$

This imples that $\left(A_{k}\right) \in w_{\mu, \beta}^{W}(p)$. Hence $L_{\infty} \cap w_{\mu, \alpha}^{W}(p) \subseteq w_{\lambda, \beta}^{W}(p)$.

Corollary 10. Let $\lambda=\left(\lambda_{n}\right)$ and $\mu=\left(\mu_{n}\right)$ be two sequences in $\Lambda$ such that $\lambda_{n} \leq$ $\mu_{n}$ for all $n \in \mathbb{N}$. If (3.3) holds, then

(a) $L_{\infty} \cap w_{\mu, \alpha}^{W}(p) \subseteq w_{\lambda, \beta}^{W}(p)$ for $0<\alpha \leq 1$, 
(b) $L_{\infty} \cap w_{\lambda, \alpha}^{W}(p) \subseteq w_{\mu}^{W}(p)$ for $0<\alpha \leq 1$,

(c) $L_{\infty} \cap w_{\lambda}^{W}(p) \subseteq w_{\mu}^{W}(p)$.

Theorem 19. Let $M$ be an Orlicz function and if $\inf _{k} p_{k}>0$, then limit of any sequence $\left(A_{k}\right)$ in $w_{\lambda, \alpha}^{W}[M, p]$ is unique.

Proof. Let $\lim _{k} p_{k}=s>0$. Suppose that $A_{k} \rightarrow A_{1}\left(w_{\lambda, \alpha}^{W}[M, p]\right)$ and $A_{k} \rightarrow$ $A_{2}\left(w_{\lambda, \alpha}^{W}[M, p]\right)$. Then there exist $\rho_{1}>0$ and $\rho_{2}>0$ such that

$$
\lim _{n \rightarrow \infty} \frac{1}{\lambda_{n}^{\alpha}} \sum_{k \in I_{n}}\left[M\left(\frac{\left|d\left(x, A_{k}\right)-d\left(x, A_{1}\right)\right|}{\rho}\right)\right]^{p_{k}}=0,
$$

and

$$
\lim _{n \rightarrow \infty} \frac{1}{\lambda_{n}^{\alpha}} \sum_{k \in I_{n}}\left[M\left(\frac{\left|d\left(x, A_{k}\right)-d\left(x, A_{2}\right)\right|}{\rho}\right)\right]^{p_{k}}=0 .
$$

Let $\rho=\max \left\{2 \rho_{1}, 2 \rho_{2}\right\}$. As $M$ is nondecreasing and convex, we have

$$
\begin{gathered}
\frac{1}{\lambda_{n}^{\alpha}} \sum_{k \in I_{n}}\left[M\left(\frac{\left|d\left(x, A_{1}\right)-d\left(x, A_{2}\right)\right|}{\rho}\right)\right]^{p_{k}} \\
\leq \frac{D}{\lambda_{n}^{\alpha}} \sum_{k \in I_{n}} \frac{1}{2^{p_{k}}}\left(\left[M\left(\frac{\left|d\left(x, A_{k}\right)-d\left(x, A_{1}\right)\right|}{\rho}\right)\right]^{p_{k} p_{k}}\right. \\
\left.+\left[M\left(\frac{\left|d\left(x, A_{k}\right)-d\left(x, A_{2}\right)\right|}{\rho}\right)\right]\right) \\
\frac{D}{\lambda_{n}^{\alpha}} \sum_{k \in I_{n}}\left(\left[M\left(\frac{\left|d\left(x, A_{k}\right)-d\left(x, A_{1}\right)\right|}{\rho}\right)\right]^{p_{k}}\right. \\
\left.\quad+\frac{D}{\lambda_{n}^{\alpha}} \sum_{k \in I_{n}}\left[M\left(\frac{\left|d\left(x, A_{k}\right)-d\left(x, A_{2}\right)\right|}{\rho}\right)\right]^{p_{k}}\right) \rightarrow 0 \text { as } n \rightarrow \infty,
\end{gathered}
$$

where $\sup _{k} p_{k}=H$ and $D=\max \left(1,2^{H-1}\right)$. Therefore we get

$$
\lim _{n \rightarrow \infty} \frac{1}{\lambda_{n}^{\alpha}} \sum_{k \in I_{n}}\left[M\left(\frac{\left|d\left(x, A_{1}\right)-d\left(x, A_{2}\right)\right|}{\rho}\right)\right]^{p_{k}}=0 .
$$

As $\lim _{k} p_{k}=s$, we have

$$
\lim _{k \rightarrow \infty}\left[M\left(\frac{\left|d\left(x, A_{1}\right)-d\left(x, A_{2}\right)\right|}{\rho}\right)\right]^{p_{k}}=\left[M\left(\frac{\left|d\left(x, A_{1}\right)-d\left(x, A_{2}\right)\right|}{\rho}\right)\right]^{s}
$$

and so $A_{1}=A_{2}$. Hence the limit is unique. 


\section{REFERENCES}

[1] R. C. Buck, "Generalized asymptotic density," Amer. J. Math., vol. 75, pp. 335-346, 1953, doi: 10.23072372456.

[2] R. Çolak, “On $\lambda$-statistical convergence,” Conference on Summability and Applications, Istanbul, Turkey, 12-13 May 2011.

[3] R. Çolak, Statistical Convergence of Order $\alpha$, Modern Methods in Analysis and Its Applications. Anamaya Pub., New Delhi, 2010.

[4] R. Çolak and C. A. Bektaş, “ $\lambda$-statistical convergence of order $\alpha$,” Acta Math. Sci., vol. 31, no. 3, pp. 953-959, 2011.

[5] A. Esi, "The A-statistical and strongly (A-p)-Cesaro convergence of sequences," Pure and Appl. Math. Sci., vol. XLIII, no. 1-2, pp. 89-93, 1996.

[6] M. Et, M. Çinar, and M. Karakaş, "On $\lambda$-statistical convergence of order $\alpha$ of sequences of function," Jour. Ineq. Appl., vol. 2013, no. 204, p. 8 pages, 2013, doi: 10.1186/1029-242x-2013-204.

[7] M. Et, S. A. Mohiuddine, and A. Alotaibi, "On $\lambda$-statistical convergence and strongly $\lambda$-summable functions of order $\alpha$, Jour. Ineq. Appl., vol. 2013, no. 469, p. 8 pages, 2013, doi: 10.1186/1029242x-2013-469.

[8] H. Fast, "Sur la convergence statistique," Coll. Math., vol. 2, pp. 241-244, 1951.

[9] J. A. Fridy, "On statistical convergence," Analysis, vol. 5, no. 4, pp. 301-313, 1985.

[10] A. D. Gadjiev and C. Orhan, "Some approximation theorems via statistical convergence," Rocky Mt. J. Math., vol. 32, no. 1, pp. 129-138, 2012, doi: 10.1216/rmjm/1030539612.

[11] B. Hazarika, "Wijsman Orlicz asymptotically ideal $\phi$-statistical equivalent sequences," Journal of Function Spaces and Applications, vol. 2013, pp. Article ID 257 181, 9 pages, 2013, doi: $10.1155 / 2013 / 257181$.

[12] B. Hazarika and A. Esi, "On $\lambda$-asymptotically Wijsman generalized statistical convergence of sequences of sets," Tatra Mountains Mathematical Publications-Number Theory, vol. 56, pp. 6777, 2013, doi: 10.2478/tmmp-2013-0025.

[13] B. Hazarika and A. Esi, "Statistically almost $\lambda$-convergence of sequences of sets," European Journal of Pure and Applied Mathematic, vol. 6, no. 2, pp. 137-146, 2013.

[14] B. Hazarika, A. Esi, and N. L. Braha, "On asymptotically Wijsman lacunary $\sigma$-statistical equivalent set sequences," Journal of Mathematical Analysis, vol. 4, no. 3, pp. 33-46, 2013.

[15] L. Leindler, "Über die la Vallée-Pousinsche Summierbarkeit Allgemeiner Orthogonalreihen," Acta Math. Acad. Sci. Hung., vol. 16, pp. 375-387, 1965, doi: 10.1007/bf01904844.

[16] J. Lindenstrauss and L. Tzafriri, “On Orlicz sequence spaces,” Israel Jour. Math., vol. 10, pp. 379-90, 1971.

[17] M. Mursaleen, “ $\lambda$-statistical convergence," Mathematica Slovaca, vol. 50, no. 1, pp. 111-115, 2000.

[18] F. Nuray and B. E. Rhoades, "Statistical convergence of sequences of sets," Fasciculi Mathematici, vol. 49, pp. 1-9, 2012.

[19] W. H. Ruckle, "FK spaces in which the sequence of coordinate vectors is bounded," Canad. Jour. Math., vol. 25, pp. 973-978, 1973, doi: 10.4153/cjm-1973-102-9.

[20] T. Salát, "On statistically convergent sequences of real numbes," Math. Slovaca, vol. 30, pp. 139_ 150,1980

[21] I. J. Schoenberg, “The integrability of certain functions and related summability methods," Amer. Math. Monthly, vol. 66, pp. 361-375, 1959, doi: 10.2307/2308747.

[22] H. Steinhaus, "Sur la convergence ordinaire et la convergence asymptotique," Coll. Math., vol. 2, pp. 73-74, 1951.

[23] R. A. Wijsman, "Convergence of sequences of convex sets, cones and functions," Bull.Amer.Math.Soc., vol. 70, pp. 186-188, 1964, doi: 10.1090/s0002-9904-1964-11072-7. 
[24] R. A. Wijsman, "Convergence of sequences of convex sets, cones and functions II," Trans.Amer.Math.Soc., vol. 123, no. 1, pp. 32-45, 1966, doi: 10.2307/1994611.

[25] A. Zygmund, Trigonometrical Series, vol. 5 of Monografías de Matemáticas, Warszawa-Lwow, 1935 .

[26] A. Zygmund, Trigonometric Series, Cambridge Univ. Press, Cambridge, UK, 1979.

\section{Authors' addresses}

B. Hazarika

Department of Mathematics, Rajiv Gandhi University, Rono Hills, Doimukh-791 112, Arunachal Pradesh, India

E-mail address: bh_rgueyahoo.co.in

\section{A. Esi}

Adiyaman University, Department of Mathematics, 02040 Adiyaman, Turkey

E-mail address: aesi23@hotmail.com 